\title{
INTERPOLATION SERIES FOR INTEGRAL FUNCTIONS OF EXPONENTIAL TYPE
}

\author{
BY \\ A. J. MACINTYRE
}

1. Introduction. This paper investigates interpolation series for integral functions $F(z)$ of exponential type from the Laplace representation

$$
F(z)=\frac{1}{2 \pi i} \int_{\Gamma} f(\zeta) e^{z \zeta} d \zeta
$$

in a manner similar to that given recently by R. C. Buck [4]. In his account the factor $e^{z \zeta}$ is expanded into a series of the form $\sum_{0}^{\infty} u_{n}(z) g_{n}(\zeta)$ and a series for $F(z)$ of the form $\sum_{0}^{\infty} u_{n}(z) T_{n}(F)$ results from (1.1) on term by term integration. In the present paper the factor $f(\zeta)$ is expanded in a series of the form $\sum_{0}^{\infty} T_{n}(F) v_{n}(\zeta)$ and the same series as before then appears for $F(z)$. The two methods give in broad outline similar results, but there are some properties which are exhibited better or even only by one of the two methods. Thus Buck's methods appear to be more powerful in dealing with Abel's series. The modified forms given by Schoenberg [15] for the Lidstone and Whittaker two point series however do not appear in Buck's analysis. They appear quite naturally below not only for the Lidstone and Whittaker series but also for the analogous Poritsky and Gontcharoff $n$-point series. Summability properties of these modified series are also exhibited clearly just as summability properties of the original series are exhibited in the work of Buck. The basis of the present paper suggested by the problem introduced in [18] is the close relation between the functions

$$
f(z, \alpha)=\sum_{0}^{\infty} F^{(n)}(\alpha) z^{-n-1}
$$

for different values of $\alpha$. (Cf. [11, 590 (b); 3].) Now the interpolation series of Gontcharoff or Poritsky are based on certain of the values $F^{(n)}\left(\alpha_{p}\right)$ assumed to be known. This information is expressed below in terms of the functions $f\left(z, \alpha_{p}\right)$ and leads to a simple functional equation for $f(z)$ and hence to an interpolation series for $F(z)$. On certain assumptions $f(z)$ will be defined apart from poles whose residues then appear as undetermined constants. When all poles are excluded by conditions of growth imposed on $F(z)$ one obtains the classical interpolation series. When all but a finite number of poles are so excluded one obtains series analogous to those given by Schoenberg for the two point expansions. These series justify uniqueness theorems sharper than

Received by the editors October 20, 1952. 
those derived from convergence or summability of the original interpolation series. It is easy to modify them further so as to obtain series which are convergent for any integral function without restriction as to order [cf. 1; 9]. There results an explicit solution to a problem solved as to existence by Pólya [12].

When the same methods are applied to Abel's series relating to the determination of $F(z)$ by the sequence of values $F^{(n)}(n)$, the results are less complete since more difficult functional equations are involved.

A final section compares certain special series and shows that summability properties have a certain consistency not always enjoyed by the convergence properties.

2. Notations and formulae of transformation. $F(z)$ will throughout represent an integral function of exponential type (at most) so that a representation of the type $(1.1)$ is valid. The functions $F(z)$ and $f(z)$ are connected by the integral relations

$$
\begin{aligned}
& f(z)=z^{-1} \int_{0}^{\infty} e^{-t} F\left(t z^{-1}\right) d t=\int_{0}^{\infty} e^{-t z} F(z) d t \\
& F(z)=\frac{1}{2 \pi i} \int_{\Gamma} e^{z \zeta} f(\zeta) d \zeta
\end{aligned}
$$

and alternatively by means of the power series representations

$$
F(z)=\sum_{0}^{\infty} a_{n} z^{n} / n !, \quad f(z)=\sum_{0}^{\infty} a_{n} z^{-n-1} .
$$

The function $f(z, \alpha)$ of (1.2) is evidently the Laplace transform of $F(z+\alpha)$ and

$$
\begin{aligned}
f(z, \alpha) & =\int_{0}^{\infty} e^{-t z} F(t+\alpha) d t=\int_{\alpha}^{\infty} e^{-(t-\alpha) z} F(t) d t \\
& =e^{\alpha z}\left\{f(z)-\int_{0}^{\alpha} e^{-t z} F(t) d t\right\}=e^{\alpha z} f(z)-\Phi(z, \alpha)
\end{aligned}
$$

where $\Phi(z, \alpha)$ is an integral function. It follows that $e^{-\alpha z} f(z, \alpha)-f(z)$ is also an integral function and that

$$
F(z)=\frac{1}{2 \pi i} \int_{\mathbf{r}} e^{(z-\alpha) \zeta} f(\zeta, \alpha) d \zeta
$$

(2.2) and (2.3) are of course effectively the Laplace formula (2.1) for $F(z+\alpha)$.

The growth function of $F(z)$ is defined as usual by

$$
h(\theta)=h(\theta, F)=\lim \sup r^{-1} \log \left|F\left(r e^{i \theta}\right)\right| .
$$


We use the notation $K\{H(\theta)\}$ for the class of all integral functions of exponential type with $h(\theta, F)<H(\theta)[4$, p. 283].

$h(-\theta)$ is necessarily the supporting function of a convex set $D$ or $D(F)$ of points in the complex plane.

We have [11, pp. 581-585] $h(\theta)=\max _{z \in D} \Re\left(z e^{i \theta}\right)$. When $H(-\theta)$ is chosen as the supporting function of the rectangle of vertices $\pm a \pm b i$ we write $K(a, b)$ in place of $K\{H(\theta)\}$ [4, p. 284]. $f(\zeta)$ is regular outside $D(F)$ and $\Gamma$ in $(2.1)$ can be any curve enclosing $D(F)[11$, p. 585, Satz 3]. From (2.2) it follows that $f(z, \alpha)$ is also regular outside $D(F)$.

3. Interpolation of Poritsky type. We shall describe as Poritsky's problem [14] that of determining $F(z)$ from the set of values

$$
F^{(k m)}\left(a_{p}\right) \quad(k=0,1,2, \cdots ; p=1,2, \cdots, m) .
$$

A knowledge of this set of values does not give any of the functions $f\left(z, a_{p}\right)$ immediately but does lead to a set of equations which they must satisfy. Let us write $\omega=e^{2 \pi i / m}$ and have

$$
\begin{aligned}
\psi\left(z, a_{p}, m\right) & =\sum_{n=0}^{\infty} F^{(n m)}\left(a_{p}\right) z^{-n m-1}=\frac{1}{m} \sum_{k=0}^{m-1} \omega^{k} f\left(\omega^{k} z, a_{p}\right) \\
& =\frac{1}{m} \sum_{k=0}^{m-1} \omega^{k} \exp \left(a_{p} \omega^{k} z\right)\left(f \omega^{k} z\right)+\frac{1}{m} \sum_{k=0}^{m-1} \omega^{k} \Phi\left(\omega^{k} z, a_{p}\right) .
\end{aligned}
$$

With $p=1,2, \cdots, m$ this can be regarded as a set of $m$ equations to determine the $m$ functions $f\left(\omega^{k} \boldsymbol{z}\right)$. Writing

$$
\frac{1}{m} \sum_{k=0}^{m-1} \omega^{k} \Phi\left(\omega^{k} z, a_{p}\right)=\Psi\left(z, a_{p}, m\right)
$$

we have

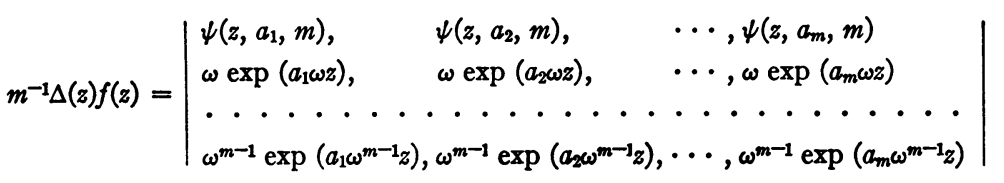

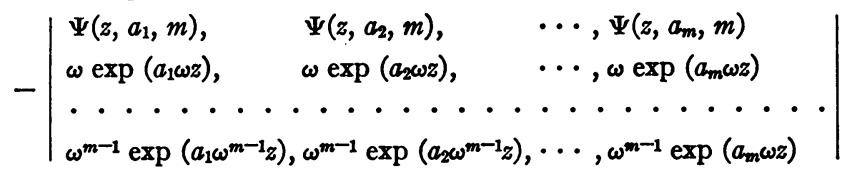

where

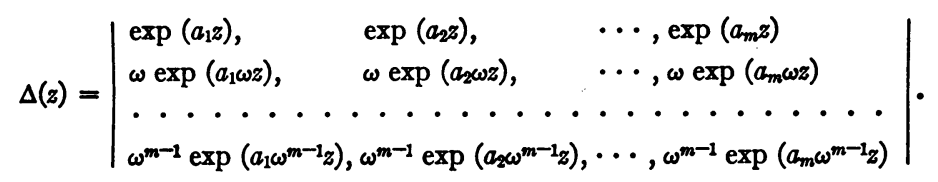

We must note that $\Delta(z)$ has a zero of order $m(m-1) / 2$ at the origin and 
that the second determinant in (3.4) has a zero of at least this order( $\left.{ }^{1}\right)$. The coefficient of $z^{N}$ in the expansion $\omega^{-m(m-1) / 2} \Delta(z)$ is clearly the sum over all sets of non-negative integers $p_{1}, p_{2}, \cdots, p_{m}$ such that $p_{1}+p_{2}+\cdots+p_{m}=N$ of the determinants

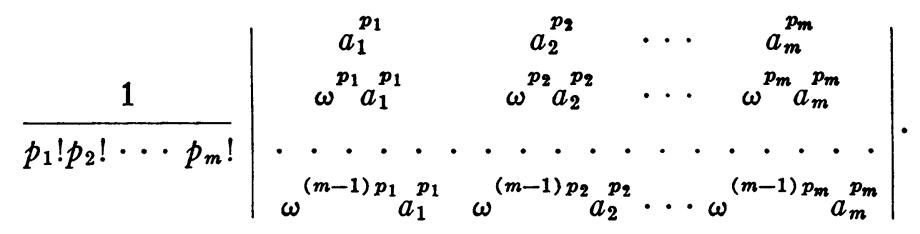

The determinant (3.6) will vanish unless $p_{1}, p_{2}, \cdots, p_{m}$ are all different, and hence if $N$ is less than $m(m-1) / 2$. The coefficient of $z^{m(m-1) / 2}$ is given by

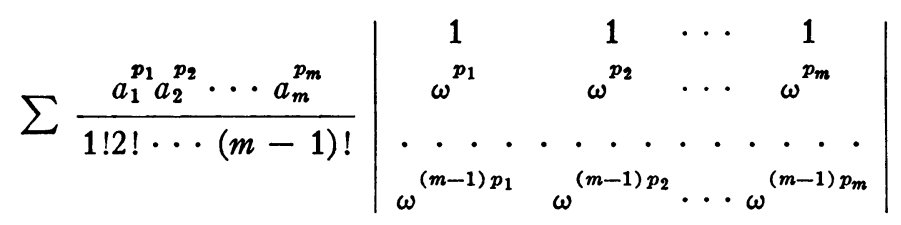

where the sum is taken over all permutations $p_{1}, p_{2}, \cdots, p_{m}$ of $0,1,2, \cdots$, $m-1$. Now the determinant in (3.7) changes sign by the interchange of $p_{r}$ and $p_{s}$ and has therefore the values $\pm \Delta$ according as $p_{1}, p_{2}, \cdots, p_{m}$ is an even or odd permutation of $0,1,2, \cdots, m-1$ where

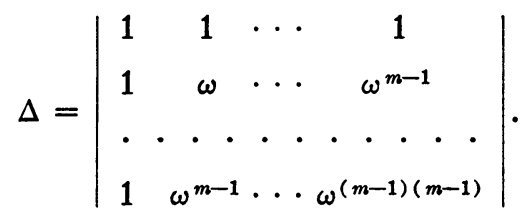

The required coefficient is therefore

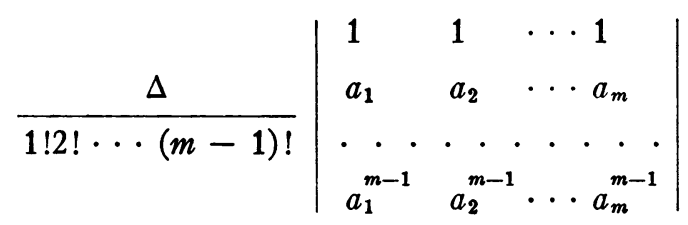

and is not zero. From (3.3), $\psi\left(z, a_{p}, m\right)$ has a zero of order $m-1$ at least, and expanding the second determinant of (3.4) in terms of its first row, we see that each minor has a zero of order $(m-1)(m-2) / 2$ from the result just proved. The determinant itself therefore has a zero of order $(m-1)(m-2) / 2$ $+m-1=m(m-1) / 2$. It now follows from (3.6) that $f(z)$ is determined by the functions

(1) The following proof of these assertions was provided by Mr. F. W. Ponting. 


$$
\psi\left(z, a_{1}, m\right), \psi\left(z, a_{2}, m\right), \cdots, \psi\left(z, a_{m}, m\right)
$$

of (3.2) except for the addition of meromorphic function whose poles are at the zeros of $\Delta(z)$ other than the origin. It follows from the Laplace transform (1.1) that $F(z)$ is determined by the set of values (3.1) apart from a function of the form

$$
\sum P_{k}(z) e^{\alpha_{k} z}
$$

the sum being taken over certain of the zeros $\alpha_{k}$ of $\Delta(z)$. Each $P_{k}(z)$ is a polynomial whose degree is less than the order of the zero $\alpha_{k}$.

If the type $\Lambda$ of $F(z)$ is less than the modulus of the smallest zero (other than $z=0)$ of $\Delta(z)$ it is evident that the sum (3.8) vanishes. Take $\Gamma$ to be a circle $|\zeta|=R$ with $R>\Lambda$ but such that $|\zeta| \leqq R$ contains no zero of $\Delta(\zeta)$ other than the origin. $f(\zeta)$ may be replaced in (1.1) by

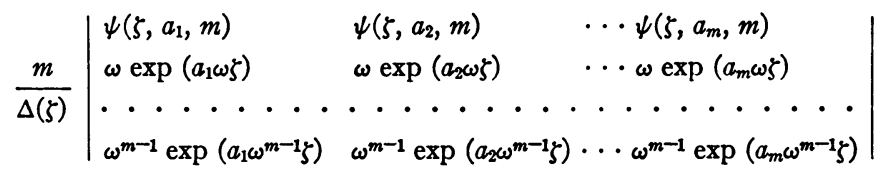

and on expanding each $\psi\left(\zeta, a_{p}, m\right)$ in powers of $\zeta$ a polynomial expansion

$$
F(z)=\sum_{p=1}^{m} \sum_{n=0}^{\infty} F^{(n m)}\left(a_{p}\right) P_{n, p}(z)
$$

results. This is effectively the result of Poritsky [14, p. 277]. We may clearly add the following remarks:

(a) If $f(\zeta)$ is regular in an inverse Mittag-Leffler star including all zeros of $\Delta(\zeta)$ other than 0 , then $[8$, p. 79$]$

$$
f(\zeta)=\lim _{\alpha \rightarrow+0} \sum_{0}^{\infty} \frac{a_{n} \zeta^{-n-1}}{\Gamma(1+n \alpha)}
$$

in the interior of the star, and there is a similar representation for each of the functions $f\left(\zeta, a_{p}\right)$. From the Laplace integral (1.1) using a contour $\Gamma$ within the star but excluding all zeros of $\Delta(\zeta)$ except the origin, we then have

$$
F(z)=\lim _{\alpha \rightarrow+0} \sum_{p=1}^{m} \sum_{0}^{\infty} F^{(n m)}\left(a_{p}\right) P_{n, p}(z) / \Gamma(1+n \alpha) .
$$

Conditions on $F(z)$ which ensure that $f(\zeta)$ is regular in a given Mittag-Leffler star are not familiar but at a cost of using a convex region instead such conditions are available. If $\Omega$ is the Mittag-Leffler star formed by excluding all radii from zeros of $\Delta(\zeta)$ other than the origin to infinity and if $D(F)$ lies within $\Omega$, then (3.10) holds [4, p. 296].

(b) If the values (3.1) all vanish, then the first determinant on the right of (3.4) vanishes and it is clear that the only functions of exponential type 
for which this occurs are the finite sums (3.8). This result generalises that of Schoenberg [15] for the case $m=2$.

(c) For functions $F(z)$ of type $\Lambda, f(\zeta)$ is regular for $|\zeta|>\Lambda$. The circle $|\zeta| \leqq \Lambda$ will contain only a finite number of zeros of $\Delta(\zeta)$. Take $\Gamma$ to be a circle $\zeta \mid=R$ with $R>\Lambda$ but such that $|\zeta| \leqq R$ contains no more zeros of $\Delta(\zeta)$ than $|\zeta| \leqq \Lambda$.

On replacing $f(\zeta)$ in (1.1) by the expression (3.9) and adding the appropriate sum of the form (3.8) we still obtain the function $F(z)$. Writing $\psi_{p}^{*}(\zeta)$ for the cofactor of $\psi\left(\zeta, a_{p}, m\right)$ in (3.9) we have

$$
\left.F(z)=\sum_{\left|\alpha_{k}\right| \leqq \Lambda} P_{k}(z) z^{\alpha_{k} z}\right)+\sum_{p=1}^{m} \frac{1}{2 \pi i} \int_{|\zeta|=R} \psi\left(\zeta, a_{p}, m\right) \stackrel{*}{*}(\zeta) e^{z \zeta} d \zeta .
$$

Expanding each $\psi\left(\zeta, a_{p}, m\right)$ in powers of $\zeta$ there results

$$
F(z)=\sum_{\left|\alpha_{k}\right| \leqq \Lambda} P_{k}(z) e^{\alpha_{k} z}+\sum_{p=1}^{m} \sum_{n=0}^{\infty} F^{(n m)}\left(a_{p}\right) Q_{n, p}(z) .
$$

The functions $Q_{n, p}(z)$ defined by

$$
Q_{n, p}(z)=\frac{1}{2 \pi i} \int_{|\zeta|=R} \zeta^{-n-1} \psi_{p}^{*}(\zeta) e^{z \zeta} d \zeta
$$

are no longer polynomials since $\psi_{p}^{*}(\zeta)$ will have poles at the zeros of $\Delta(\zeta)$. But they will differ from the polynomials $P_{n, p}(z)$ only by exponential sums of the type (3.8). $Q_{n, p}(z)$ can be considered as the remainder in an expansion asymptotic as $n$ tends to infinity for $P_{n, p}(z)$. The expansion (3.11) has been given by Schoenberg [15] in the case $m=2$. Our derivation exhibits summability properties similar to those described in (a). Our results can be summed up in

THEOREM 1. An integral function $F(z)$ of type $\Lambda$ possesses the expansion (3.11), the infinite series being convergent. If $E$ be the set of $\alpha_{k}$ such that $D(F)$ intersects the radius from $\alpha_{k}$ to infinity, then

$$
F(z)=\sum_{\alpha_{k} \ni B} P_{k}(z) e^{\alpha_{k} z}+\sum_{p=1}^{m} \sum_{n=0}^{\infty} F^{(n m)}\left(a_{p}\right) Q_{n, p}(z)
$$

where the functions $Q_{n, p}(z)$ are defined by (3.12), and the infinite series are understood to be summed by the method of Mittag-Leffer as in (3.10).

4. Interpolation of Gontcharoff type. Gontcharoff's problem relating to the determination of $F(z)$ by the sequence

$$
F\left(a_{1}\right), F^{\prime}\left(a_{2}\right), \cdots, F^{(m-1)}\left(a_{m}\right), F^{(m)}\left(a_{1}\right), F^{(m+1)}\left(a_{2}\right), \cdots, F^{(2 m)}\left(a_{1}\right) \cdots
$$

is from our point of view very similar to that of Poritsky. The sequence (4.1) defines the functions 


$$
\begin{aligned}
\chi\left(z, a_{p}, m, p\right) & =\sum_{n=0}^{\infty} F^{(n m+p-1)}\left(a_{p}\right) z^{-n m-p} \\
& =\frac{1}{m} \sum_{k=0}^{m-1} \omega^{p k} f\left(\omega^{k} z, a_{p}\right) \\
& =\frac{1}{m} \sum_{k=0}^{m-1} \omega^{p k} \exp \left(a_{p} \omega^{k} z\right) f\left(\omega^{k} z\right)+\frac{1}{m} \sum_{k=0}^{m-1} \omega^{p k} \Phi\left(\omega^{k} z, a_{p}\right) .
\end{aligned}
$$

These equations for $p=1,2, \cdots, m$ may be regarded as a system of $m$ equations for the functions $f\left(\omega^{k} z\right), k=0,1,2, \cdots, m-1$. The determinant of this system replacing $\Delta(z)$ is

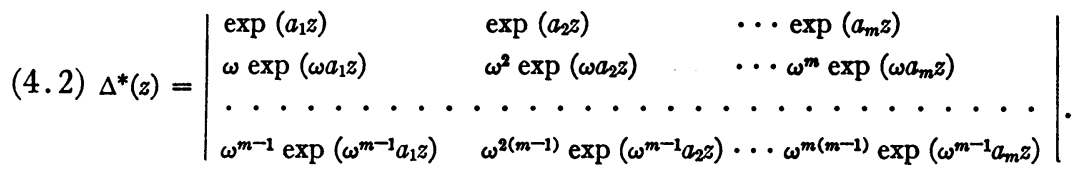

$\Delta^{*}(z)$ has no zero at $z=0$, so that the complications arising in Poritsky's problem on account of the zero of $\Delta(z)$ at $z=0$ do not arise here. If the type $\Lambda$ of $F(z)$ is less than the modulus of the smallest root $\Delta^{*}(z)$ and the contour $\Gamma$ in (2.1) is chosen as a circle $|\zeta|=R$ such that all roots of $\Delta^{*}(z)$ are outside it, then we may replace $f(\zeta)$ by

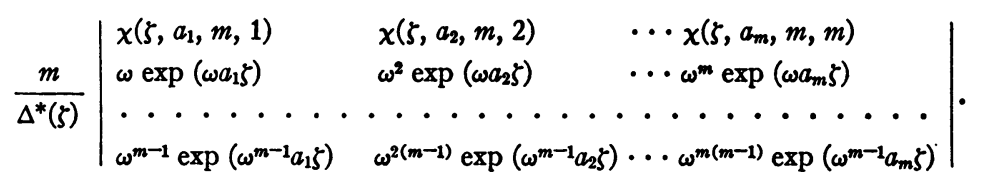

On expanding the functions $\chi\left(\zeta, a_{p}, m, p\right)$ in powers of $\zeta^{-1}$ and integrating term by term there results a polynomial expansion of the form

$$
F(z)=\sum_{p=1}^{m} \sum_{n=0}^{\infty} F^{(n m+p-1)}\left(a_{p}\right) G_{n, p}(z) .
$$

This is effectively the result of Gontcharoff [7, pp. 36-37]. We may add the remarks:

(a) If the convex region with indicatrix $H(\theta)$ contains the origin but no zero of $\Delta^{*}(z)$, and if $F(z)$ belongs to $K\{H(\theta)\}$, then the series (4.3) is summable by Mittag-Leffler's method, that is

$$
F(z)=\lim _{\alpha \rightarrow+0} \sum_{p=1}^{m} \sum_{n=0}^{\infty} F^{(n m+p-1)}\left(a_{p}\right) G_{n, p}(z) / \Gamma(1+n \alpha) .
$$

(b) When the series (4.4) holds the conclusion $F(z) \equiv 0$ follows from the vanishing of the sequence (4.1). This is a stronger result than that deduced from the convergence of (4.3).

(c) For any function of exponential type $\Lambda$ there will be an expansion of the form 


$$
F(z)=\sum_{p=1}^{m} \sum_{n=0}^{\infty} F^{(n m+p-1)}\left(a_{p}\right) H_{n, p}(z)+\sum_{\left|\alpha_{k}\right| \leqq \Lambda} P_{k}(z) e^{\alpha_{k} z}
$$

where the $H_{n, p}(z)$ are the remainders in an asymptotic expansion of the polynomials $G_{n, p}(z)$. The second sum is finite, the $P_{k}(z)$ being polynomials and the $\alpha_{k}$ being zeros of $\Delta^{*}(z)$.

(d) The series (4.5) will have summability properties similar to those discussed in remark (a) for the series (4.3).

We summarise these results in

THEOREM 2. An integral function $F(z)$ of type $\Lambda$ possesses the expansion (4.5), the infinite series being convergent. If $E$ be the set of $\alpha_{i}$ such that $D(F)$ intersects the radius from $\alpha_{i}$ to infinity, then

$$
F(z)=\sum_{\alpha_{k} \ni E} P_{k}(z) e^{\alpha_{k} z}+\sum_{p=1}^{m} \sum_{n=0}^{\infty} F^{(n m+p-1)}\left(a_{p}\right) H_{n, p}(z)
$$

with the infinite series summed by the method of Mittag-Leffler.

5. Uniqueness theorems. It follows from Theorem 1 or Theorem 2 that the only integral functions of exponential type for which the values (3.1) or (4.1) all vanish are the exponential polynomials of the kind (3.8). Now such exponential polynomials have very special growth properties and if they are excluded by a suitable condition we obtain uniqueness conditions. For example we have the following

THEOREM 3. If $D$ is a convex domain containing no zero of $\Delta(z)$ other than $z=0$ (or no zero of $\Delta^{*}$ ) in its exterior, then the integral function $F(z)$ of exponential type is uniquely determined by the values (3.1) or (4.1) if it satisfies

$$
\lim \inf \left|F\left(r e^{i \theta}\right)\right| \exp \{-H(\theta, D) r\}=0 .
$$

The condition (5.1) need only be imposed for particular values of $\theta$, one for each zero on the boundary of $D$ (cf. [2, p. 15]).

6. Existence problems. Generalised series. A problem closely related to the Lidstone expansion is the following. If $a_{n}$ and $b_{n}$ are two given sequences is it possible to satisfy the relations

$$
F^{(2 n)}(-1)=a_{n}, \quad F^{(2 n)}(1)=b_{n}
$$

by means of an integral function $F(z)$ ? This problem has been completely solved. The conditions that $\sum_{0}^{\infty} a_{n} z^{2 n} /(2 n)$ ! and $\sum_{0}^{\infty} b_{n} z^{2 n} /(2 n)$ ! are integral functions which are clearly necessary for the solution of either part of (6.1) separately are sufficient for the simultaneous solution of both parts $[17, \mathrm{p}$. 51]. The corresponding problem for the Whittaker series has a similar solution [16]. We show that the same is true for the generalised versions arising from Poritsky's problem and Gontcharoff's problem. Our method is similar to that of Appell and Hurwitz $[1 ; 9]$. It will be enough to give the details for 
one of the two cases. We consider the equations

$$
F^{(n m)}\left(a_{p}\right)=A_{n, p} \quad(p=1,2, \cdots, m ; n=0,1,2, \cdots) .
$$

The process for the construction of a solution can be summarised as follows. We take the expansion (3.13), omit the finite sum and replace the coefficients $F^{(n m)}\left(a_{p}\right)$ by the required values $A_{n, p}$. If the series converges the construction of $F(z)$ is complete, but this will be the case only if all the power series $\sum_{0}^{\infty} A_{n, p} z^{m n} /(m n)$ ! are integral functions of exponential type. To deal with the general case when these power series are assumed to represent integral functions without restriction as to order, we take the sequence of functions $Q_{n, p}(z)$ to have an order increasing with $n$ in place of a fixed order. With $p=1$ we see that

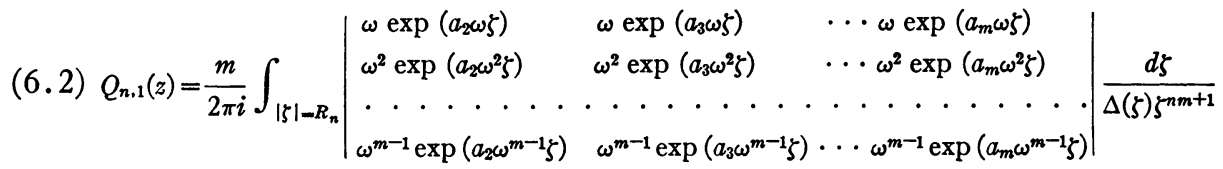

and $R_{n}$ is a suitable increasing sequence. Note first that $\Delta(\zeta)$ will be a sum of exponentials and for large $|\zeta|$ will be dominated by one or two terms (depending on $\arg \zeta$ ) of the form $A e^{\alpha \zeta}+B e^{\beta \zeta}$. It is clear then that a sequence $R_{n}$ will exist such that $n<R_{n}<n+1$ and such that $|\Delta(\zeta)|>\delta \exp \left(-C R_{n}\right)$ $(\delta>0, c>0)$ on the circle $|\zeta|=R_{n}$. It follows that, if the sequence $Q_{n, 1}(z)$ is formed with this $R_{n}$ in (6.2), then

$$
\left|Q_{n, 1}(z)\right|<\frac{K \exp \{(n+1)|z|+K n\}}{n^{m n}} .
$$

From this inequality it is clear that convergence of $\sum_{0}^{\infty} A_{n, 1} z^{n m} /(m n)$ ! for all $z$ implies convergence of

$$
\sum_{0}^{\infty} A_{n, 1} Q_{n, 1}(z)
$$

for all $z$ (uniformly in any bounded domain). Now in the expression (6.2) we see that to replace the variable $\zeta$ by $\omega \zeta$ does not affect $d \zeta / \zeta^{n+1}$, effects a cyclic permutation of the rows of the determinant $\Delta(\zeta)$, and replaces the determinant in the numerator by a cyclic permutation of another minor of $\Delta(\zeta)$. It is evident then that we may replace $(6.2)$ by

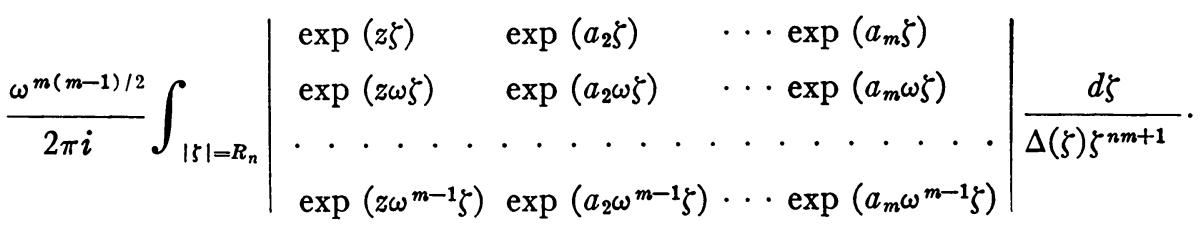

From this representation we see that $Q_{n, 1}^{(n m)}\left(a_{1}\right)=1$ and that $Q_{n, 1}^{(k m)}\left(a_{p}\right)=0$ un- 
less $p=1$ and $k=n$. If $\sum_{0}^{\infty} A_{n, 1} z^{n m} /(n m)$ ! is an integral function, then

$$
F_{1}(z)=\sum_{0}^{\infty} A_{n, 1} Q_{n, 1}(z)
$$

is also an integral function and satisfies the relations

$$
F_{1}^{(n m)}\left(a_{1}\right)=A_{n, 1}, \quad F_{1}^{(n m)}\left(a_{p}\right)=0, \quad \quad p \neq 1 .
$$

With $Q_{n, p}(z)(p=2,3, \cdots, m)$ defined in a similar manner we see that if all the series $\sum_{0}^{\infty} A_{n, p} z^{n m} /(n m)$ ! represent integral functions, then the series

$$
F(z)=\sum_{p=1}^{m} \sum_{n=0}^{\infty} A_{n, p} Q_{n, p}(z)
$$

converges to an integral function which satisfies the relations

$$
F^{(n m)}\left(a_{p}\right)=A_{n, p} \text {. }
$$

The existence theorem without the series (6.3) for a more general problem was given by Pólya [12].

7. Abel's series. The results for Abel's series are not as complete as those given by Buck [4, pp. 292-293] but will be discussed briefly. The method is to investigate the relation between $f(z)$ as defined in (2.1) and the function

$$
\psi(z)=\sum_{0}^{\infty} F^{(n)}(n) z^{-n-1} .
$$

Provided $z$ is sufficiently large we have from (2.1) the integral representation

$$
\begin{aligned}
\psi(z) & =\frac{1}{2 \pi i} \int_{\Gamma} f(\zeta) \sum_{0}^{\infty} \zeta^{n} e^{n \zeta} z^{-n-1} d \zeta \\
& =\frac{1}{2 \pi i} \int_{\Gamma} \frac{f(\zeta) d \zeta}{z-\zeta e^{\zeta}} .
\end{aligned}
$$

With $z=w e^{w}$ there follows

$$
\psi\left(w e^{w}\right)=\frac{1}{2 \pi i} \int_{\Gamma} \frac{f(\zeta) d \zeta}{w e^{w}-\zeta e^{\zeta}} .
$$

This will hold if for instance $R(w)$ is sufficiently large. If now the zero $\zeta=w$ but no other zero of $w e^{w}-\zeta e^{\zeta}$ as a function of $\zeta$ falls inside $\Gamma$, then (7.1) must be modified to

$$
\psi\left(w e^{w}\right)=\frac{1}{2 \pi i} \int_{\Gamma} \frac{f(\zeta) d \zeta}{w e^{w}-\zeta e^{\zeta}}+\frac{f(w)}{(1+w) e^{w}} .
$$

If $\Gamma$ is interior to a region in which $\zeta e^{\zeta}$ is schlicht (and hence excludes $\zeta=-1$ ) 
it follows that

$$
\psi\left(w e^{w}\right)-\frac{f(w)}{(1+w) e^{w}}
$$

is regular in and on $\Gamma$ and hence that

$$
F(z)=\frac{1}{2 \pi i} \int_{\Gamma} e^{z \zeta} e^{\zeta} \psi\left(\zeta e^{\zeta}\right)(1+\zeta) d \zeta .
$$

We now expand $\psi\left(\zeta e^{\zeta}\right)$ in a power series in powers of $\zeta e^{\zeta}$ considering either convergence or summability. We obtain for the series

$$
\sum_{0}^{\infty} F^{(n)}(n) \frac{1}{2 \pi i} \int_{\Gamma} e^{(z-n)}(1+\zeta) \zeta^{-n-1} d \zeta=\sum_{0}^{\infty} F^{(n)}(n) z(z-n)^{n-1 / n !}
$$

which is Abel's series for $F(z)$. Convergence of this series to $F(z)$ follows if $\Gamma$ can be taken inside the pear-shaped region in which $\left|\zeta e^{\zeta}\right|<e^{-1}$, and MittagLeffler summability if $\Gamma$ can be taken inside the parabolic region in which $\left|\arg \left(\zeta e^{\zeta}+1\right)\right|<\pi$. Convergence to $F(z)$ follows for all functions of $K\{\chi(\theta)\}$, $\chi(\theta)$ being the supporting function of the pear-shaped region. This property is known to hold for a wider class [4, p. 293]. For Mittag-Leffler summability the conditions $h(\theta)<\chi^{*}(\theta), h(0)<\infty$, where $\chi^{*}(\theta)$ is the supporting function of the parabolic region, suffice. This is the same condition as was obtained by Buck [4, p. 293].

8. Comparison of some special cases. Gontcharoff remarks [7, p. 38] that when $a_{1}, a_{2}, \cdots, a_{m}$ are in arithmetic progression the determinants $\Delta(z)$ and $\Delta^{*}(z)$ defined in (3.5) and (4.2) can be factorised and all their zeros expressed in elementary terms. The expansion problem can then be answered in a more explicit manner. For example if $a_{p}=p-1$, Gontcharoff's expansion is convergent for all integral functions of type less than

$$
\frac{\pi}{m \sin (\pi / m)}
$$

Now if $m$ is large this critical type is approximately unity. The behaviour might be expected for large $m$ to be similar to that of Abel's series. Here the critical type for convergence is however $\tilde{\omega}=.278 \cdots$ so that in this respect convergence properties of Abel's series do not appear as a limiting form of convergence properties of Gontcharoff's series. For Mittag-Leffler summability however the critical type for this special Gontcharoff series is still given by (8.1) and that for Abel's series is unity.

The situation with Poritsky's series in the special case $a_{p}=p-1$ is similar. The determinant $\Delta(z)$ contains the factor

$$
\exp \left(\omega^{p} z\right)-\exp \left(\omega^{q} z\right)
$$


and the smallest zero of this factor other than zero is of modulus $2 \pi /\left|\omega^{p}-\omega^{q}\right|$. If $n$ is even this modulus takes its minimum when $\left|\omega^{p}-\omega^{q}\right|=2$ and if $m$ is odd when $\left|\omega^{p}-\omega^{q}\right|=2 \cos (\pi / 2 m)$. Thus if $m$ is even the critical type in this special case for convergence of Poritsky's series is $\pi$ while if $m$ is odd the critical type is $\pi \sec (\pi / 2 m)$. The "three point" expansion with $m=3$ and critical type $2 \pi / 3^{1 / 2}$ appears as the most powerful. For large $m$ the critical type is approximately $\pi$ and the series might be compared with the GregoryNewton series which determines $F(z)$ by the sequence $F(0), F(1), F(2), \cdots$. In this case the critical type for convergence is $\log 2$ and the critical type for summability is $\pi[17$, p. $52 ; 4$, p. 288]. Similarly the critical type for convergence of the Newton-Gauss series associated with $F(0), F(-1), F(1)$, $F(-2), F(2), F(-3), \cdots$ is $\log \left(3+2\left(2^{1 / 2}\right)\right)$ while that for summability is $\pi$ [3, p. $162 ; 4$, pp. 290-291].

Another simple special problem is that of the determination of $F(z)$ by the sequence $F(0), \quad F^{\prime}(0), \cdots, F^{(m-2)}(0), \quad F^{(m-1)}(1), \quad F^{(m)}(0), \quad F^{(m+1)}(0), \cdots$ which is an instance of Gontcharoff's problem with $a_{1}=a_{2}=\cdots=a_{m-1}=0$, $a_{m}=1$. The critical type for convergence (or summability) is easily seen to be the modulus of the smallest zero of the function

$$
1+\frac{z^{m}}{m !}+\frac{z^{2 m}}{(2 m) !}+\cdots \text {. }
$$

Now this function has zeros only when $z^{m}$ is negative $[13$, pp. 67,254$]$ and as $m$ increases the modulus of the smallest zero increases and for large $m$ is approximately $(m !)^{1 / m} \sim m e^{-1}$. Hence the critical type becomes large with $m$ and shows a certain analogy with Taylor's series which may be considered as the limiting case with $m=\infty$.

\section{REFERENCES}

1. P. Appell, Sur les fonctions périodiques de deux variables, J. Math. Pures Appl. (4) vol. 7 (1891) pp. 157-219.

2. R. P. Boas, $A$ note on functions of exponential type, Bull. Amer. Math. Soc. vol. 47 (1941) pp. 750-754.

3. T. A. Brown, On a class of factorial series, Proc. London Math. Soc. (2) vol. 23 (1925) pp. 149-171.

4. R. C. Buck, Interpolation series, Trans. Amer. Math. Soc. vol. 64 (1948) pp. 283-298.

5. R. D. Carmichael, Summation of functions of a complex variable, Ann. of Math. (2) vol. 34 (1933) pp. 349-378.

6. W. Gontcharoff, Recherches sur les dérivées successives des fonctions analytiques. Genéralisation de la série d' Abel, Ann. Ecole. Norm. vol. 47 (1930) pp. 1-78.

7. - Détermination des fonctions entières par interpolation, Paris, 1937.

8. G. H. Hardy, Divergent series, Oxford University Press, 1949.

9. A. Hurwitz, Sur l'intégrale fini d'une fonction entière, Acta Math. vol. 20 (1897) pp. 285312.

10. G. J. Lidstone, Notes on the extension of Aitken's theorem (for polynomial interpolation) to the Everett types, Proceedings of the Edinburgh Mathematical Society (2) vol. 2 (1930) pp. 16-19. 
11. G. Poblya, Untersuchungen über Lücken und Singularitäten von Potenzreihen, Math. Zeit. vol. 29 (1929) pp. 549-640.

12. - Sur l'existence de fonctions entières satisfaisant a certaines conditions linéaires, Trans. Amer. Math. Soc. vol. 50 (1941) pp. 129-139.

13. G. Pólya and G. Szegö, A ufgaben und Lehrsätze aus der Analysis. II, 1925.

14. H. Poritsky, On certain polynomial and other approximations to analytic functions, Trans. Amer. Math. Soc. vol. 34 (1932) pp. 274-331.

15. I. J. Schoenberg, On certain two-point expansions of integral functions of exponential type, Bull. Amer. Math. Soc. vol. 42 (1936) pp. 284-288.

16. J. M. Whittaker, On Lidstone's series and two point expansions of analytic functions, Proc. London Math. Soc. (2) vol. 36 (1934) pp. 451-469.

17. - Interpolatory function theory, Cambridge, 1933.

18. E. M. Wright, The asymptotic expansion of integral functions and of the coefficients in their Taylor series, Trans. Amer. Math. Soc. vol. 64 (1948) pp. 409-438.

The University of AberdeEn, Aberdeen, Scotland. 